\title{
Effect of Basal Metabolic Rate on Blood Pressure among the Hill Wanchos of Arunachal Pradesh, India
}

\author{
John Basumatary ${ }^{1}$ and Gulrukh Begum ${ }^{2}$ \\ ${ }^{l}$ ICMR-SRF, Department of Anthropology, Gauhati University \\ ${ }^{2}$ Associate Professor, Department of Anthropology, Gauhati University
}

\begin{abstract}
Wanchos are one of the prominent tribe of North-east India. They are primarily hill dwellers and are mostly located in the Southern part of Arunachal Pradesh and in the South-Eastern border areas of Assam. This study focuses on the prevalence and as well as the risk of hypertension among the adult Wanchos. In this paper, it is also intended to study the relationship of Basal Metabolic Rate with blood pressure, and if this relationship is dependent on body size. 318 adult Wanchos (177 males and 141 females) ranging from 20 to 60 years of age were measured for height, weight, BMR, skinfold measurements and blood pressure. Pearson's correlation and coefficient test and linear regression analysis were used to establish any relationship between the proposed dependent and independent variables with other confounding variables. Data analysis was done in SPSS version 16.0. Considerably higher percentages of males (41.24\% and 19.77\%) were found to be in prehypertensive and hypertensive categories than females $(31.91 \%$ and $14.18 \%)$. The variance among the three blood pressure categories between the adult male and female Wanchos was found to be statistically significant $\left(x^{2}=7.0883\right.$ and $p$-value 0.028893$)$ at 0.05 level. $16.38 \%$ of males and $10.64 \%$ of females were found to be at stage 1 hypertensive stage. Pearson's correlation coefficient test showed a significant correlation between BMR and BMI, BMR and BFP (body fat percentage), BMR and systolic blood pressure, and BMR and diastolic blood pressure. However, linear regression showed a very weak association between BMR and systolic blood pressure and BMR and diastolic blood pressure. Among the adult Wanchos, Basal Metabolic Rate was not found to be a significant predictor of the variability in their blood pressure, hence no association with the prevalence of hypertension among them. Elevated Basal Metabolic Rate among the Wanchos is not resulting in their elevated blood pressure as BMR is significantly correlated with their daily energy expenditure or metabolic equivalent (MET) per day. Basal Metabolic Rate is negatively associated with the MET per day, implying that as the intensity of physical activity increases among the adult Wanchos, their resting energy expenditure (or BMR) decreases.
\end{abstract}

Keywords: adult; association; energy expenditure; hypertensive; significant. 